\title{
¿TIENE LUGAR LA RECREACIÓN EN LA ESCUELA? \\ LAS LUDOTECAS COMO ESTRATEGIA
}

\author{
DOES RECREATION TAKE PLACE AT SCHOOL? THE TOY LIBRARY LIKE STRATEGY
}

Esperanza Osorio Correa ${ }^{1}$

Resumen

Este artículo plantea en principio la pregunta sobre el lugar de la recreación en la escuela y la posición que el campo de la recreación deberá plantearse en algún momento, más aún cuando en nuestro país se están formando licenciados en recreación que obligan a pensar la praxis misma en el ámbito escolar. En segundo lugar propone a las ludotecas escolares como una opción posible para generar de manera intencional procesos de educación para la recreación en la escuela, en tanto éstas se resignifiquen para el contexto escolar, lo que demanda problematizar una institución -la ludoteca- que se ha instaurado como una estrategia para la garantía y protección del derecho al juego y la recreación.

Palabras clave: campo, recreación, ludoteca, lúdica, juego.

Abstract

This article pose at first, the question on the place of the recreation in the school and the position that the field of the recreation will have to appear in some moment, even more when in our country they are formed licensed in recreation that they force to think the practice itself about the school area. Secondly it proposes the school toy library as a possible option to generate in an intentional way processes of education for the recreation in the school while these re-mean for the school context what demands questioning an institution -the play library- that has been establish as strategy for the guarantee and protection of the right to the game and the recreation.

Key words: field, recreation, toy library, ludic, game.

Fecha de recepción: 30 de Septiembre de 2010

Fecha de aprobación: 29 de Octubre de 2010

\footnotetext{
${ }^{1}$ Directora de funlibre. Psicóloga Universidad Nacional de Colombia. Especialista en Gerencia Social de la Escuela de Administración Pública - ESAP. Magister en Desarrollo Social y Educativo Cinde de la Universidad Pedagógica Nacional. Correos: espeosorio@funlibre.org - espeosorio@gmail.com
} 
El artículo 67 de la Constitución Política afirma: "la educación es un servicio público que tiene una función social [...]. La educación formará al colombiano [...] en la práctica del trabajo y la recreación, para el mejoramiento cultural, científico, tecnológico y para la protección del ambiente. El Estado, la sociedad y la familia son responsables de la educación [...]", significa esto que ¿la recreación debe tener un lugar en la escuela? Preguntas como la anterior han derivado en múltiples reflexiones sobre la educación y han definido un conjunto de aspectos en los modos de aproximarse al análisis de los conceptos y prácticas de la recreación.

Estas reflexiones, soportadas por múltiples miradas, gradualmente nos han planteado la posibilidad de un marco de análisis que orienta la toma de posición de la recreación como campo (Bourdieu, 1985). Este horizonte da la oportunidad de avanzar en la tarea de superar dicotomías, lugares comunes y retóricas que aunque han sido parte de la construcción misma del campo, en la medida que están imbricadas en el pensamiento cotidiano, han determinado una visión simplificada de la recreación, que pasa por alto su aproximación como un conjunto de prácticas, que se relacionan no sólo con las actividades y técnicas, sino con problemas fundamentales del campo, como sus conceptos, tránsitos, agentes, construcciones en la sociedad contemporánea y sus complejas interacciones con el desarrollo humano.

En el campo de la recreación existen múltiples dicotomías, entre ellas, aquellos que la ubican como un modelo de negocios o un modelo de justicia social, o la tensión entre la recreación como parte del deporte y la educación física y no como un campo, aunque relacionado con estas categorías, autónomo, e incluso desde lo institucional su dependencia del Ministerio de Educación Nacional al Ministerio de Cultura. Así el denso entramado de relaciones que vincula preguntas sustanciales con otras perspectivas o áreas del conocimiento.

Este artículo no pretende establecer definiciones, busca problematizar el campo de la recreación como un lugar en la escuela, a través de un objeto de análisis específico: la ludoteca escolar. Esto permite ponerse en perspectiva, es decir, ubicar en un horizonte de sentido en el que la lucha de fuerzas y tensiones antes que ignorarlas se hacen evidentes y demandan una toma de posición, acerca del lugar de la recreación en la escuela.

Lúdica pedaqóq. Vol 2, No. 15 (ene- dic. 2010) pp. 6- 16
Dado que en el campo de la recreación la tradición académica es un proceso apenas en desarrollo, por lo menos en nuestro país, el riesgo de orientar sus prácticas a partir de fórmulas convencionales, que si bien tienen su antecedente en múltiples experiencias y prácticas significativas, no se han consolidado o incluso agrupado como un objeto de estudio común. Este escenario genera que las prácticas estén saturadas de lugares comunes que se repiten, y donde se toman como punto de partida conceptos de la norma o investigadores que son recurrentes sin un acercamiento crítico que lo sitúe en discusiones más actuales.

Desde esta perspectiva la recreación afronta grandes desafíos y espacios por ocupar, como el hecho de posicionarse como una práctica que hace parte de las representaciones y experiencias de los seres humanos, un campo académico científico, como lo es cualquier "disciplina" con su propio desarrollo, identidad y ámbito de actuación.

Parte de los "paradigmas" convencionales y limitantes que se generan por la mirada a la recreación desde lugares comunes, es que la escuela no sea identificada como una institución relevante para el campo. Se le asigna un espacio como una de las tantas opciones de los programas de tiempo extraescolar, que en muchos de los casos se reduce a los objetivos de una educación industrial² (De Zubiría, 2006), donde en reducidas ocasiones se reconoce el valor del impacto social y educativo en lo psicológico, lo social, como estrategia para aumentar la calidad de la educación y su papel en la formación para la vida.

En este contexto el campo de la recreación y sus agentes, o quienes por tener una relación de proximidad con él, por ejemplo los educadores físicos, tienen el imperativo de problematizar y abordar la complejidad y la construcción de aproximaciones y marcos de análisis de la recreación como objeto de estudio, sin evitar las tensiones y conflictos que se trasladan en ocasiones "al patio trasero" por la comodidad que implica sumarse a la doxa políticamente establecida desde arriba. Esto exige debatir, dialogar, pero principalmente actuar, lo que implica

2 Se reconocen la inclusión explícita por parte de la Secretaría de Educación del Distrito del tema en el programa Escuela Ciudad Escuela. 
decisión, elección y una apuesta por el campo, que como Morín (1999) señala "en la noción de apuesta existe la conciencia de riego y de incertidumbre".

El campo de la recreación, como otros en emergencia, pareciera verse impuesto cada vez que se aborda su discusión, de volver de manera reiterada a su historia e incluso a la lucha permanente por hacer reconocer el derecho a su existencia legítima y así mismo su reconocimiento como derecho, la gran mayoría de las veces está presente la situación de tener que argumentar su sentido, solicitando atención sobre el campo y exigiendo un espacio, delimitando sus límites distintivos.

Se asume como parte de un ejercicio necesario el reivindicar su posición crítica, ya que gana autonomía y legitimación frente al descuido de la sociedad y el desinterés de otros campos de conocimiento políticos y de poder, a una industria del entretenimiento y unas lógicas de consumo, y por otra parte, hacia las prácticas y creencias de agentes del campo que se constituyen en tendencias autodestructivas y de fragmentación.

Es así como un gran número de textos e investigaciones deben iniciar su argumentación, retomando sus relaciones con el ocio griego, el ocio romano, el surgimiento y visibilización del término como derivación de las reivindicaciones del tiempo libre en el llamado periodo de la industrialización y la reflexión crítica sobre su dependencia con los procesos productivos, sólo por mencionar de manera bastante reducida sus antecedentes.

Sin embargo, es importante avanzar en la conceptualización y que mejor para ello que tomar resultados de investigaciones que dan cuenta de los conceptos de recreación de las personas en concreto para analizarlo.

En investigaciones realizadas en el marco de contratos de ludotecas, experiencia que se inició en el año 1990 con el diseño de una ludoteca itinerante para los hogares comunitarios del Instituto Colombiano de Bienestar Familiar (ICBF) hasta los proyectos realizados en el año $2010^{3}$, la tendencia en rela-

3 En la página de la fundación: www.funlibre.org, se encuentra la experiencia asociada directamente al tema de las ludotecas desde el año 1990, con instituciones como el ICBF, ción con los conceptos de recreación de niños, niñas, jóvenes y adultos mayores muestran una regularidad en las categorías emergentes, aunque obviamente con diferencias propias de la edad y de los contextos que no se discutirán aquí.

El empezar por este breve análisis de las representaciones sociales en relación con la recreación, responde al necesario reconocimiento que debemos hacer sobre la influencia que estos tienen en las posibilidades de gestión y desarrollo. Como señala Ibáñez (1994) una representación social es pensamiento constituido y pensamiento constituyente, en el primer caso, éstas se transforman en productos a partir de los cuales se interpreta la vida social, en el segundo, la representación social se hace parte del objeto que representa. Un análisis de contenido de los textos recopilados permiten identificar como categorías emergentes: actividad, tiempo libre, diversión, placer, libertad, salud, bienestar, salir de la rutina, entre otras, y con una menor presencia la experiencia. En general, se observa la construcción de conceptos de recreación vinculados a la vida cotidiana de los sujetos (lo que hacen con los amigos y la familia, por ejemplo), y a una oferta de recreación institucional estandarizada. Por otra parte, se evidencia particularmente en los adultos una relación de la recreación funcional, así se expresa comúnmente en los textos que ésta contribuye a la salud mental y física de los individuos.

En un diagnóstico realizado en el marco del contrato con la Secretaría de Educación del Distrito en el año 2004, con la participación de 30 instituciones educativas de 13 localidades y 1362 personas entre maestros, directivos, estudiantes y padres de familia. El mayor porcentaje de respuestas se acumulan en la opción: la recreación como una actividad (48\%) y como una experiencia que lo hace sentir bien (28.5\%). En relación con las prácticas se encontró que la tendencia se da hacia los juegos al aire libre en docentes y estudiantes. Sin embargo los datos evidencian que en general se practican juegos diversos, en los grupos de administrativos y de padres de familia predomina la participación en juegos seguida por la participación en juegos de mesa. Los

OIM, Centros de Convivencia, Corporación Día del Niño, Fundación Sala de Asilos, Comnfamiliar Afidro - ICBF, ludotecas escolares con la Secretaría de Educación del Distrito y con la OIT, proyecto Espacios Lúdicos con Coldeportes. 
docentes y el grupo administrativo expresan que los estudiantes prefieren jugar en el colegio, seguido por el parque. Los padres de familia presentan una tendencia a jugar usualmente en el parque seguido por la casa. En general las respuestas apuntan al uso de diferentes lugares para jugar, en donde el parque, el colegio y la casa predominan.

La mayoría de las definiciones de recreación la focalizan como una forma de actividad. Kraus, citado por Edginton et al. (1998), presenta varias definiciones que han sido desarrolladas a través de los años y que son una representación de la realidad -que aunque limitada- da cuenta de cómo se configura como pensamiento constituido:

La recreación está ampliamente relacionada con la actividad (física, mental, social o de compromiso emocional) en contraste con la ociosidad o el completo descanso. La recreación puede incluir un amplio rango de actividades tales como deportes, juegos, música, teatro, viajes, pasatiempos y actividades sociales. Estas actividades pueden ser realizadas esporádicamente o de manera sostenida, una sola vez o a través de la vida. La elección de la actividad o la participación es completamente voluntaria y no se debe a presiones externas. La recreación es estimulada por motivación interna y el deseo por obtener satisfacción personal, más que el logro de propósitos ulteriores u otros objetivos o recompensas extrínsecas. La recreación tiene una fuerte dependencia de un estado de la mente o actitud; esta no es el propósito de la actividad sino más bien la razón para hacerla y la forma como los sentimientos individuales acerca de ella lo que hace la actividad recreacional. La recreación tiene resultados potenciales deseables, a través de la motivación primaria para la participación puede lograr disfrute personal y puede resultar en crecimiento físico, intelectual y social. Esto no quiere decir que la recreación es deseable automáticamente; existen actividades que son peligrosas, indeseables, degenerativas para la personalidad. Sin embargo cuando se realizan como parte de un programa de recreación comunitaria se asume que la recreación está diseñada para promover actividades constructivas.

La conceptualización de la recreación con su par inseparable, el tiempo libre, que aquí se denomina: el tiempo de ocio, ha mostrado un mayor movimiento en función de prácticas emergentes derivadas del desarrollo de la tecnología y las comunicaciones, en las que es necesario profundizar con estudios de lar- go alcance. Sin embargo, se evidencia la valoración que las personas hacen de la recreación como una experiencia psicológica y social.

Aquí la recreación se asume como una estrategia mediadora en la "provocación" de "vivencias" que involucran procesos psicológicos y sociales, donde entran en interacción el sujeto (con su historia, sus significados subjetividades), los cuales se ponen en escena a partir de la relación con los otros y con el entorno. Tanto el tiempo histórico como el actual, cobran vida en el espacio de la vivencia, haciéndola dinámica y vital.

Una vivencia tiene múltiples lecturas de los valores, comportamientos e historias que se tejen en un sólo espacio, en el cual el juego que se establece flexibiliza barreras y hace a los participantes más humanos y genuinos propiciando el autoconocimiento y el conocimiento del otro, el aprendizaje, el logro de metas y una relación de igualdad entre quienes participan en la medida que se crea un contexto donde todos los elementos y dimensiones se funden en la vivencia.

En este sentido planteo que la recreación no únicamente está dada para la diversión o el consumo de productos de entretenimiento sino que en sí misma favorece la creación de espacios para el desarrollo humano y por ende ha de ocupar un lugar en la vida cotidiana de los sujetos.

Por sus dimensiones psicológica y social, una vivencia implica al sujeto mismo, involucra la puesta en interacción de las dimensiones afectiva, cognitiva, social, afectiva, comunicativa, física, estética y espiritual de los seres humanos, que se traducen en su desarrollo en un tiempo y un espacio que le atribuye características particulares a los sujetos o grupos.

La vivencia de la recreación se hace significativa cuando posibilita salir de los lugares comunes, de lo predeterminado, de lo constituido, cuando nos pone en contacto con la vida y permite preguntarse sobre la vida misma, cuando no es sólo consumo de prácticas y objetos determinados por la lógica del mercado.

Una mirada a la recreación en su complejidad, multidimensionalidad y carácter polisémico, le obligan a construirse desde la transdisciplinariedad, y si 
bien recoge conceptos y métodos de otros campos, deberá establecer sus bordes y sus propios modos de producir conocimiento para el campo, además, salir de la situación de subalternidad en que se le ha colocado en otras disciplinas, expresada por ejemplo, en la ausencia en currículums de formación de profesionales en las ciencias sociales y educativas, o escasa presencia en la formación de los educadores físicos, con consecuencias claras en su invisibilización histórica y actual en la escuela.

Es por ello, que el avanzar en propuestas conceptuales y metodológicas que contribuyan a dar pistas de los modos a través de los cuales la recreación puede ocupar un lugar significativo en la escuela es de importancia. En este sentido se considera que las ludotecas, pueden ser una estrategia integradora por superar los vacíos y limitaciones de los sistemas educativos y enriquecer los procesos y experiencias en el aula.

Desde esta perspectiva, en primer lugar, es necesario reconocer dos vacíos que existen en la escuela en relación con la recreación, por una parte, se ignora (la recreación rompe el orden, evidencia la diversidad, la tradición, reconoce intereses y talentos particulares), y por otra, la limita a eventos y celebraciones específicas. Como consecuencia hay que reconocer las limitaciones para el posicionamiento de metodologías como las ludotecas en la escuela. El que éstas existan, así sea en un número reducido en las instituciones educativas, no quiere decir que su "utilidad" sea reconocida y apropiada como perteneciente al campo de la recreación, lo que demanda especificar entre otros aspectos, qué hace diferente una ludoteca escolar de otras instituciones similares por ejemplo en el ámbito comunitario.

\section{Las ludotecas}

Estudios realizados muestran que la ludoteca más que como un medio para favorecer procesos de educación para la recreación y el tiempo libre, son consideradas principalmente un instrumento didáctico para los procesos de aprendizaje (SED-FUNLIBRE, 2004), y aunque en menor medida, pero no por ello menos importante, un medio para la potenciación de la dimensión lúdica de los estudiantes y el "aprovechamiento de la recreación y el tiempo libre", por otra parte, le atribuyen funciones relacionadas con el desarrollo de habilidades, la convivencia, el desarrollo humano, entre otras.
Las ludotecas históricamente se han definido como un lugar o institución creada para generar oportunidades de recreación y tiempo libre a los niños y las niñas a partir del préstamo de juguetes, orientaciones para jugar. A partir de la revisión de diferentes autores se ha encontrado que la ludoteca se define principalmente como un lugar o una institución donde principalmente se garantiza y protege el derecho de los niños y las niñas al juego, a la recreación y al juguete y definiciones donde se relaciona con la educación para el ocio y el tiempo libre.

Desde una perspectiva convencional, la ludoteca en la escuela tiene entre sus principales objetivos: posibilitar que la educación sea menos formal, más integradora y volcada hacia el desarrollo humano integral; posibilitar a todos los niños y niñas el acceso al juego como una forma de socializar su uso, permitiendo especialmente un contacto amplio y diverso con el mismo; valorar el acto de jugar respetando la libertad, la iniciativa, la creatividad, la autonomía, posibilitando una formación de autoconcepto positivo; rescatar la sensibilidad y el juego; compartir momentos de alegría; proporcionar un espacio de aprendizaje, educación en valores, desarrollo de competencias y habilidades, entre otros (Silva da Paz, 2002).

Una de las definiciones más usuales sobre la ludoteca es considerarla una institución recreativocultural pensada para niños, niñas y adolescentes con el fin de desarrollar su personalidad a través del juego y el juguete. Construidas en centros comunitarios, escuelas, bibliotecas, casas de cultura y/o instituciones socioculturales que posibilitan, favorecen y estimulan el juego infantil ofreciendo materiales y orientaciones para el juego (Borja, 1980), lugares para la socialización, la integración social, la construcción de representaciones infantiles (Pires dos Santos, 2002).

Borja (2002), afirma que lo que diferencia las ludotecas de otras instituciones es su objetivo central de desarrollar actividades lúdicas a partir del préstamo de juguetes y materiales de juego.

Las ludotecas se conciben como espacios diseñados a la medida del niño, donde pueden explorar y aprender a vivir, puede estar en la escuela, o por fuera de la misma pero articulada al sistema educativo ofreciendo asistencia técnica y alternativas de 
actividades libres y planificadas a los estudiantes. Lo importante es que le dé al niño la posibilidad de jugar en la medida de su deseo y necesidad proporcionando al estudiante condiciones más favorables para su formación.

En general las ludotecas son definidas desde tres elementos fundamentales: el espacio, el juego y sus beneficios e implicaciones para el desarrollo integral del niño, no se concibe como un lugar aislado en la vida de los niños y las niñas sino estrechamente articulado a la escuela y a la familia.

Desde esta perspectiva la ludoteca es un espacio humano y escenario para el desarrollo, donde la condición del sujeto estudiante interactúa con los elementos del medio, los maestros y el juguete construyendo interacciones que le posibilitan el disfrute y a la vez ganar en aprendizaje para la vida. Más allá de la búsqueda de competencias técnicas, la ludoteca es un "lugar" donde se aprende sobre las relaciones, los valores, se experimenta y descubre dentro de un marco de libertad, por ello no puede correr el riesgo de entrar en los esquemas formales de enseñanza, ha de ser innovadora y cambiante.

Como sucede con otras instituciones éstas se flexibilizan a la luz de las realidades y contextos concretos donde son instauradas. En Colombia particularmente, entidades públicas, ONGs, cajas de compensación, entre otras, han puesto en marcha proyectos de ludotecas que pretenden responder a problemáticas específicas de la infancia que habita los lugares donde son instaladas.

La rapidez de las transformaciones sociales y las realidades socioculturales, generan un cambio permanente en los contextos de desarrollo del niño y de la niña, lo que ha hecho que se modifiquen las formas y los fines que persiguen las ludotecas. También surge como respuesta a las problemáticas del hogar, a los problemas de convivencia, y a las pocas opciones con que cuentan los niños y niñas para disfrutar de tiempo de ocio y recreación y en general de espacios para el juego y la diversión (Dinello, 2000).

Un avance en el modelo de las ludotecas como lo plantea Dinello, es el papel que los niños y niñas han de jugar en la ludoteca, tema de una vigencia significativa a la luz de sus derechos y particularmente del derecho a la participación (a ser escuchado, a que sus opiniones sean tenidas en cuenta, a participar en las discusiones sobre los asuntos que los afectan, a tener acceso a la información, a organizarse, etc., como se consagra en la Convención de los Derechos del Niño).

La necesidad de responder a problemáticas específicas de la infancia, ha hecho de las ludotecas una estrategia privilegiada, sin embargo, se corre el riesgo de vaciarse de contenido en la medida que en sus aplicaciones se configuran como una solución orientada a la carencia y no al desarrollo. Esto es, que se asuma como un medio para ofrecer respuestas compasivas o paliativas, o como reparadora de los mismos, más que cumplir con su propósito esencial de ofrecer un espacio para el desarrollo y para garantizar el ejercicio del derecho al juego y la recreación por parte de los niños, niñas y adolescentes con propósitos ligados a la vida y la realidad.

Desde esta perspectiva, la ludoteca no es sólo un espacio para el juego sino en su dimensión más amplia para la recreación. Como señalan McLean y Brattain (2008) el ocio, la recreación y el juego son categorías estrechamente relacionadas:

[...] el ocio por ejemplo, provee las oportunidades para el juego y la recreación; mucho del tiempo libre en la sociedad moderna es absorbido por la recreación. El ocio puede incluir actividades de educación continuada, prácticas religiosas, o servicios comunitarios los cuales usualmente no se dan a través de formas de recreación. Aunque el juego y la recreación tienden a superponerse, no son lo mismo. El juego no es tanto una actividad como una forma de conducta. Puede ocurrir durante el trabajo o el ocio, mientras la recreación tiene lugar únicamente durante el tiempo de ocio. La recreación obviamente incluye muchas formas de juego, pero también puede involucrar otras actividades como viajar, leer, ir a museos, e involucrar otras actividades culturales o intelectuales. Como institución social la recreación tiene un rango amplio de aplicaciones. De los tres términos, es uno de los más comprensibles y significativos para las personas. Es más fácilmente reconocida como un área de actividad personal y responsabilidad social y sus valores son evidentes para todos los grupos de edad y las comunidades.

La amplia gama de opciones y prácticas asociadas a la recreación, la enfrenta a nuevos y complejos cuestionamientos acerca de su capacidad para res- 
ponder a las necesidades propias de su desarrollo como campo, por ejemplo, los problemas de la sociedad actual: la exposición a información amplia y diversa de todos los géneros (política, científica, cultural, desastres, ideologías), mayor atención a las consideraciones éticas en virtud de prácticas que ponen en riesgo a las personas, la influencia de los medios de comunicación y de la internet y sus efectos particulares sobre las prácticas de recreación y tiempo libre (Rojeck, 2005). El ritmo de los cambios sociales, las realidades socioculturales, transforman constantemente el contexto de desarrollo de los niños, niñas y jóvenes. De ahí que veamos día a día un crecimiento de problemáticas que se evidencian en el ámbito escolar. La institución educativa, por lo tanto, se encuentra en la obligación de ofrecer espacios de interacción en los cuales se fome a los estudiantes para enfrentar los desafíos de la sociedad actual.

En tanto organismo vivo toda comunidad está permanentemente definiendo qué conserva de sí y qué transforma. Uno de los lugares privilegiados en donde delega esta misión es la escuela. Ella recibe a sus niños y les transmite una imagen del mundo, de su naturaleza física y social, de sus jerarquías y tradiciones. Les enseña que tienen un pasado definido por las decisiones que se han tomado en otros tiempos y les señala un contexto, unos derechos y unos deberes a través de cuyo ejercicio podrán, a la larga, intervenir en esas decisiones. La enseñanza primaria los localiza en una cultura particular en un mundo general. La enseñanza secundaria los orienta a las posibles formas de insertarse en aquella. La enseñanza superior los inscribe en unas comunidades disciplinares y profesionales que determinan lo central de su existencia social (Universidad Nacional de Colombia, Facultad de Artes, Unidad de Arte y Educación, 2007, p. 26).

Nuevas y complejas realidades hacen que se presente un desfase entre la realidad y los desarrollos conceptuales del campo, lo que nos obliga a problematizar el tema de las ludotecas y su papel como estrategia por excelencia del campo, resignificarlas, superar el pensamiento predicativo y el discurso cerrado, para colocarnos en el pensamiento epistémico, es decir, "permitir que el pensamiento se pueda colocar ante las circunstancias" (Zemelman, 2004). Tal planteamiento supone no negar la práctica en nombre de una teoría, que de este modo, deja de ser teoría para ser verbalismo o intelectualismo, o negar la teoría en nombre de una práctica, que de esta forma se arriesga a perderse en torno a sí misma. $\mathrm{Ni}$ elitismo teórico ni el basismo practicista, sino la unidad teórica y práctica (Freire, 2005), riesgo que es permanente en el campo de la recreación, y en las ludotecas como estrategia derivada del campo.

Desde este marco de análisis, se plantean algunos interrogantes en relación con la ludoteca escolar: ¿las ludotecas en la escuela están limitadas como medio para acceder al juego y dar soporte a las didácticas utilizadas en el salón de clase?, ¿las ludotecas desde su definición más común y el modo como se diseñan y operan aportan a procesos de educación para la recreación?, sin pretender dar respuestas, considero que debemos ponerlo en el plano de la duda para avanzar en la construcción de propuestas integrales de desarrollo que si bien no reemplazan las funciones de la escuela, las complementa y cualifica.

En este contexto la ludoteca se ubica como un proyecto ético, estético, político y social que no se limita al juego, crea las condiciones para ofrecer espacios donde los estudiantes puedan gozar de la recreación como una vivencia. Esto señala la necesidad de ubicar la recreación y por ende a las ludotecas en el contexto contemporáneo y abrir posibilidades de acción más amplias que permitan la expresión de procesos recreativos en sus aspectos más integrales (emocionales, afectivos, cognitivos, espirituales, estéticos, prácticas culturales). Por otra parte, la ludoteca concebida como un espacio de construcción de conocimiento social, puede constituirse en oportunidades tanto para el desarrollo de los estudiantes como para el campo de la recreación.

La experiencia recreativa establece un puente dialógico con lo real, sin perder la mirada hacia mundos posibles, donde lo que "es" puede cambiar y lo que "no es" puede ser una experiencia que no es externa al sujeto, sino de la que él hace parte, además trascienden propósitos instrumentales, su horizonte de sentido lo orienta al desarrollo y a la dignidad humana como condición para estar en el mundo. Dado que no existe una teoría, aún consolidada que explique la vivencia en su totalidad para el campo de la recreación, es necesario apostarle a su construcción, y a reconocer que no toda práctica recreativa es "positiva" (adjetivación que se hace en función de la racionalidad subyacente), o que sea el medio, como se ha perpetuado, de preparar a las personas para el aparato productivo, o en el mejor de los casos, que se considere una condición secundaria en relación con el trabajo. 
La ludoteca como proyecto se configura a partir del ordenamiento y estructuración de unos elementos filosóficos, teóricos, metodológicos, humanos, institucionales que crean un contexto donde los discursos, las actividades y las acciones generan unas respuestas que no siempre están predeterminadas, pero que pretenden crear escenarios donde la exploración y el descubrimiento de nuevos modos de conocimiento sean posibles para que los estudiantes se recreen y opten por experiencias que les permitan vehiculizar talentos e intereses, en este sentido las respuestas y los aprendizajes pueden ser inesperadas y sorprendentes.

El enfoque que se ha venido construyendo a partir de la experiencia ha incorporado nuevas categorías a partir de sus aplicaciones, sistematizaciones y evaluaciones. En la gráfica 1, se muestra una síntesis de lo que hoy en día representa las categorías principales que orientan nuestros ejercicios de diseño de las ludotecas. Como síntesis es necesario aclarar que cada una de las categorías tiene en sí misma implícitas múltiples reflexiones y conceptos.
El punto de partida, y que coincide con algunos autores e investigadores en el tema de ludotecas, es que ésta es por excelencia un espacio de educación para y mediante la recreación:

La educación para la recreación vista como una estrategia que posibilita crear espacios de resistencia, empoderamiento y transformación, no implica desconocer los fines de disfrute, esparcimiento y placer de las actividades recreativas, lo que implica es tomar posición frente a las alternativas que se ofrecen y que quienes se recrean actúen con mayor libertad y autonomía en relación con el tipo de vida que quieren. Involucra que quien se recrea no es consumidor pasivo, sino que la recreación le amplia las oportunidades para renovar e innovar su visión de mundo y el lugar que quiere ocupar en él (Osorio, 2009).

Educación que trasciende las prácticas educativas convencionales, cuyos contenidos están orientados a la mediación de procesos que en lo concreto se traduzcan en el desarrollo de capacidades humanas complejas, que comprenden desde la capacidad de

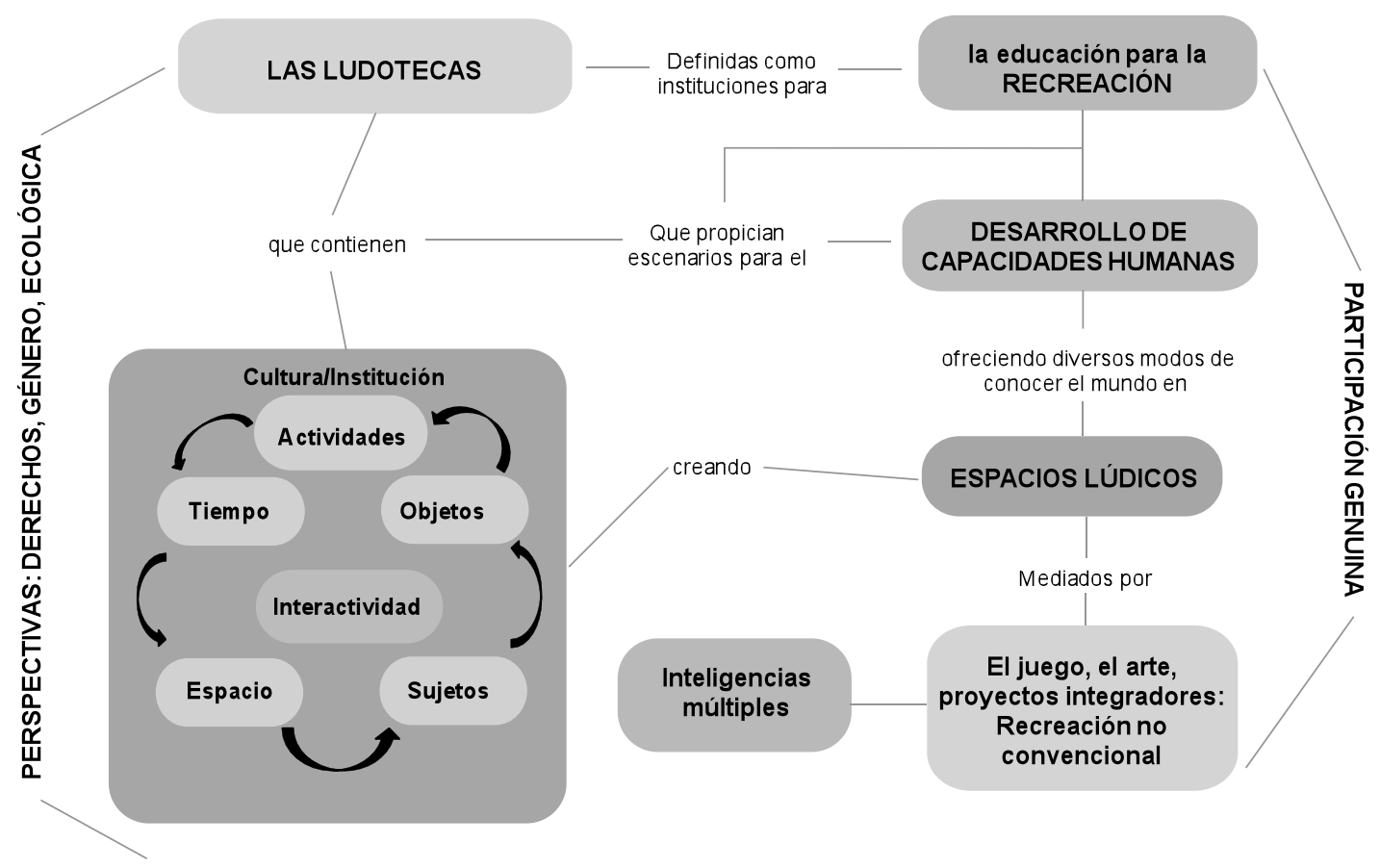

Gráfica 1: síntesis de categorías orientadoras de las ludotecas 
participación en la vida social, como la oportunidad de desarrollar talentos que se traduzcan en una mayor apropiación de los procesos de construcción de sujeto y de comprensión de la realidad, el ser capaz de platearse resistencias a poderes e injusticias reparables, por mencionar algunas. El tomar posición al ubicarse en el enfoque de capacidades humanas, significa igualmente tomarlo con cautela en sus posibilidades de construcción y pertinencia frente a nuestras propias realidades e identidades. En este sentido se considera central en su relación con la libertad, y cómo ampliar las oportunidades -mediante el empoderamiento y la resistencia- de tener el acceso a los medios para lograr la vida que se quiere. Implica por otra parte, el reconocimiento de ciertos valores fundamentales para el desarrollo y el respeto de identidades y capacidades locales.

Así las múltiples perspectivas que orientan el diseño de las ludotecas (derechos, género y ecológica) le apuestan a una mirada particular al desarrollo humano que debe ser construida con los estudiantes, $y$ una reflexión constante en la sociedad y su relación con el entorno natural y cultural.

La ludoteca como espacio lúdico se configura como un lugar "provocador" de experiencias, de desafíos, en el que intervienen un espacio físico, unos medios y unos elementos, un tiempo, que mediados y armonizados por un ludotecario(a), crean oportunidades de expresión y desarrollo para los participantes. Como lo plantea Arbeláez (2002):

El espacio lúdico hace parte de la misma situación existencial: el espacio que es un continuum socio-histórico; cultural y físico no puede ser revolucionado y enriquecido sin la activación y dinamización de la imaginación y la creatividad de los hombres y las mujeres en su encuentro colectivo y cotidiano quienes desde el ejercicio de la lúdica y la recreación en el intercambio de pensamiento e imaginación en el espacio lúdico regresarían a desordenar la casa y a redefinir el futuro para que el individuo vuelva a salir a la calle, al bulevar, el paseo, el parque la plaza a redimensionarlos y significarlos como los espacios donde habitan los otros. Donde el conflicto generado por la puesta en escena de los mismos en común puede ser dirimido de manera civilizada y humana, es decir, imaginativa y creativa.

Pensada para la escuela, se puede entender como un proyecto que vinculado a los objetivos institucio- nales mantiene su carácter propio como una opción de libertad, creatividad y experimentación, que no sigue las normas del salón de clase, sino que por el contrario, da la opción de replantear las jerarquías que limitan la participación genuina infantil y juvenil. Esto es posible en la medida que las ludotecas se reconozcan como espacios que se construyen mediante la interacción de múltiples dimensiones, donde el ludotecario(a), los objetos, lo institucional, no son considerados como variable independiente y a los estudiantes como variable dependiente, sino como un contexto donde todos hacen parte, y por lo tanto se plantean relaciones equitativas en la medida que aunque juegan un papel en el espacio, la interactividad se da por el hecho de estar ahít.

En este sentido quiero dejar un último planteamiento relacionado con el juego como categoría de lo lúdico. Anteriormente enunciaba que el juego y el juguete, desde sus concepciones clásicas, no son suficientes para decidir sobre el diseño de la ludoteca y sus elementos, dado que al ampliarlo al campo de la recreación puede abarcar otras opciones que tradicionalmente no son denominadas juego pero que podrían entrar en la categoría de lo lúdico, que se asume como característica central en lo recreativo.

A mi parecer, Haydée Silva (s.f.), en su texto Paradigmas y niveles del juego hace un llamado inteligente y desafiante al problematizar las nociones de juego en sí mismas, las cuales no profundizaré aquí pero nos permiten orientar una toma de posición frente al papel del juego y el juguete en la ludoteca.

La autora propone cuatro niveles de la metáfora lúdica: material, estructura, contexto y actitud.

Al material se refiere como el conjunto de objetos unidos entre sí por vínculos usados para jugar, es decir, que alguien les da un valor lúdico; a la estructura se refiere como un sistema de reglas que el jugador se impone para llevar a cabo su acción, sin embargo, señala que tampoco hay estructura que sea lúdica en sí, no basta un sistema de reglas para confirmar la existencia del juego. La estructura indica el esquema de la acción, más no el sentido que cabe darle. El contexto ${ }^{5}$, entendido como el conjunto de elementos

\footnotetext{
${ }^{4}$ Se toman elementos de la definición de contexto de Erikson y Shultz (1977).

${ }^{5}$ El contexto lúdico incluye factores como la determinación
} 
objetivos y/o subjetivos exteriores al juego, los jugadores y los juguetes propiamente dichos que los condicionan, es fundamental para la interpretación de los demás niveles. Por último a la actitud lúdica se refiere a la postura del sujeto frente al juego y que le da el significado y sentido en sí mismo.

Estos cuatro niveles de referencia pueden ayudar a determinar las actividades y prácticas de la ludoteca desde una mirada más amplia, donde se incluya a los estudiantes en el proceso de construcción de la ludoteca, de los juegos y de las actividades, y donde se replantee la preeminencia jerárquica del ludotecario(a), que como se dijo se apartan del carácter evaluativo o instrumental de la actividad. Ejercicio que contribuye a que el campo de la recreación avance en la definición de lo que hace sus prácticas distintivas de otros campos o actividades, y acerca de cómo avanzar en la construcción de espacios lúdicos que recreen valga la redundancia la dimensión lúdica de los estudiantes.

Crear estos espacios significa aproximarse a los modos de aprender e interactuar de los estudiantes, como anota (Gardner, 2002) existen suficientes pruebas positivas de que algunas personas adoptan una aproximación primordialmente lingüística al aprendizaje, mientras que otras prefieren un rumbo especial o cuantitativo. Igualmente algunos estudiantes obtienen los mejores resultados cuando se les pide que manejen símbolos de clases diversas, mientras que otros están mejor capacitados para desplegar su comprensión mediante demostraciones prácticas o a través de interacciones con otros individuos, los seres humanos son capaces de conocer el mundo de modos diferentes (a través del lenguaje, del análisis lógico matemático, de la representación espacial, del pensamiento musical, del uso del cuerpo para resolver problemas o hacer cosas, de una comprensión de los demás individuos y de nosotros mismos), donde los individuos se diferencian es en la intensidad de estas inteligencias y en las formas en que se

histórica (época, sociedad, sistema de valores en curso, imaginario colectivo, medio social, género, pertenencia generacional y nacional, lengua de los jugadores) y la determinación ideológica (condiciones de acceso a la cultura y al saber, acceso real, nexos con la ideología dominante, nexos entre jugadores, nexos con las instituciones). Abarca también las condiciones concretas de realización de la actividad (lugar, tiempo, entorno físico). recurre a esas mismas y se las combina para llevar a cabo diferentes labores, para solucionar problemas diversos y progresar en diversos ámbitos.

Desde esta perspectiva el proyecto "lúdico" como opción metodológica podría abrir múltiples posibilidades creativas y de apropiación de estilos de vida de recreación de los estudiantes desde la primera infancia y en virtud de sus intereses de desarrollo. Por ello la ludoteca supone un espacio donde se resignifican las relaciones, se construyen significados compartidos entre los adultos, los niños, los adolescentes y los jóvenes, y se privilegia la expresión y comunicación desde formas diversas y creativas, donde hablar, opinar y re-crear es posible.

La ludoteca está estrechamente articulada a la dinámica escolar, no es un espacio aparte de la misma, o un mecanismo administrativo para el préstamo de juguetes o la dotación de elementos para los maestros, es un espacio lúdico, una posibilidad a partir de la cual a través de las dinámicas y prácticas con los niños, las niñas, los adolescentes y los jóvenes pueden replantear o construir una nueva y más placentera relación con el conocimiento y el arte, además, la desmitificación y el redimensionamiento de las relaciones con el adulto, maestro o ludotecario, le pueden brindar la experiencia de participar en la construcción de nuevos saberes desde sus propias capacidades y de acuerdo a su edad y nivel de desarrollo.

La ludoteca escolar concebida desde el enfoque que aquí se propone, es un escenario para el desarrollo humano, es un espacio para reivindicar, defender y garantizar el derecho al juego, la recreación y la participación, coadyuvar a procesos de transformación cultural, en ella se pueden experimentar relaciones democráticas de género, intergeneracionales e institucionales que posibiliten al estudiante y a la comunidad educativa vivenciar formas de interacción más equitativas y solidarias y un espacio para identificar estados emocionales, percepciones y opiniones de los estudiantes en un ambiente más libertario y con una alta capacidad para escuchar y tener en cuenta sus opiniones e intereses, es un modo de darle un "lugar a la recreación en la escuela".

Ampliar la mirada a la ludoteca implica redimensionar sus propósitos en torno a las posibilidades que ofrece en tanto espacio de construcción de un 
conocimiento que permita a los estudiantes una mayor comprensión de los contenidos que recibe en la escuela y de la realidad misma, como espacio de formación para una ciudadanía social que involucra el desarrollo de capacidades humanas para jugar un papel activo en acciones colectivas tenientes a lograr los niveles de bienestar deseados, no a la espera de que sea adulto, sino como un proceso gradual de construcción como sujeto político y social desde la primera infancia.

Lo anterior no conlleva a desviar sus propósitos como espacio de educación para la recreación, sino por el contrario, al hacer de ésta un componente sustancial en sus procesos de formación, abre el espacio para el despliegue de potencialidades, el desarrollo de capacidades, la articulación y sinergia de actividades proyectadas al espacio extraescolar y las actividades festivas, es decir, se recoge y reconoce como categoría intencional en la escuela.

\section{Referencias}

Arbeláez, J. (2002). Espacio lúdico: una construcción social y comunitaria: caso Bogotá, Cartagena de Indias, Colombia, Memorias VII Congreso Nacional de Recreación II Elarel Vicepresidencia de la República, Coldeportes/FUNLIBRE. Obtenido el 28 de julio de 2002, desde: www.funlibre.org

Bourdieu, P. (1985). Razones prácticas sobre la teoría de la acción, Barcelona, Anagrama.

De Borja, M. (1980). El juego infantil: organización de las ludotecas, Barcelona, Ediciones Oikos-tau.

De Zubiría Samper, M. (2006). Psicología del talento, Colombia fundación internacional de pedagogía conceptual.

Dinello, R. (2000). 'Las ludotecas en la posmodernidad. Una visión latinoamericana'. En Borja Solé (Comp.) Las ludotecas. Instituciones de juego, España, Ediciones Octaedro, s.l.

Edginton, Ch., Hanson, C., Edginton, C., Hudson, S.D. (1998) Leisure programming. A service centered and benefits approach, Boston, Mcgraw-hill.

Freire, P. (2007). Pedagogía de la tolerancia, Crefa, Buenos Aires, Fondo de cultura económica.

Gardner, H. (2002). La mente no escolarizada, Barcelona, Paidós.

Ibáñez, T. (1994). La construcción del conocimiento desde una perspectiva.

Mclean, Hurd y Brattain (2008). Recreation and leisure in modern society, Jones a Bartlett publishers inc.

Morin, E. (2007). Introducción al pensamiento complejo, Barcelona, Gedisa.
Osorio, E. (2009). Consideraciones iniciales para la construcción de una propuesta de educación para la recreación. Obtenido, desde: www.funlibre.org

Pire Dos Santos, S. (2002). 'Espacios lúdicos: brinquedoteca'. En Santa Marli Pires Dos Santos (Comp.) Crianza, adulto, e o lúdico, Petrópolis: editora vozes.

Rojeck, C.(2005) Leisuretheory. Principles and practice, Palgrave Macmillan.

Silva H. (s.f.). Paradigmas y niveles del juego, Université de paris III - Sorbonnenouvelle, UNAM.

Silva Paz da Tania, R. (2002). 'Ludoteca en la escuela'. En Ludoteca o lúdico en diferentes contextos, Petrópolis, Editora vozes.

'Socioconstruccionista'. En Montero, M. (coord.). Conocimiento, realidad e ideología, Caracas, Venezuela, Avepso.

(2007). Experiencia y acontecimiento. Reflexiones sobre la educación artística, Bogotá, Universidad Nacional de Colombia, Facultad de Artes, Unidad de arte y educación. 